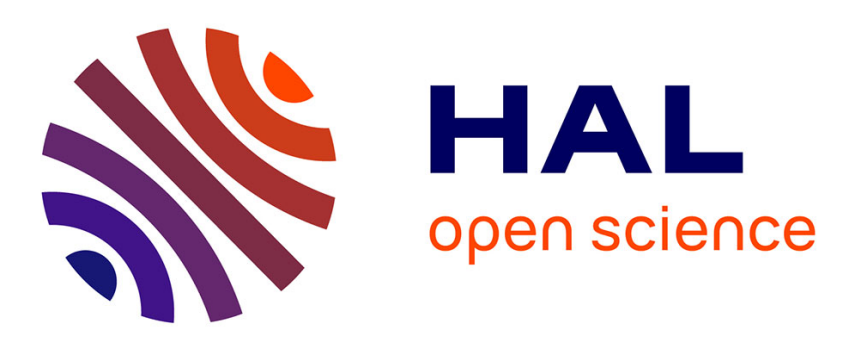

\title{
An adaptive finite element model for well-bore breakout simulation
}

Diogo L Cecílio, Philippe R B Devloo, Sonia M. Gomes, Erick S R Santos

\section{To cite this version:}

Diogo L Cecílio, Philippe R B Devloo, Sonia M. Gomes, Erick S R Santos. An adaptive finite element model for well-bore breakout simulation. 2018. hal-01858387

\section{HAL Id: hal-01858387 \\ https://hal.science/hal-01858387}

Preprint submitted on 20 Aug 2018

HAL is a multi-disciplinary open access archive for the deposit and dissemination of scientific research documents, whether they are published or not. The documents may come from teaching and research institutions in France or abroad, or from public or private research centers.
L'archive ouverte pluridisciplinaire HAL, est destinée au dépôt et à la diffusion de documents scientifiques de niveau recherche, publiés ou non, émanant des établissements d'enseignement et de recherche français ou étrangers, des laboratoires publics ou privés. 


\title{
An adaptive finite element model for well-bore breakout simulation
}

\author{
Diogo L. Cecílio \\ Universidade de São Paulo \\ Philippe R. B. Devloo \\ Universidade Estadual de Campinas \\ Sônia M. Gomes \\ Universidade Estadual de Campinas \\ Erick S. R. Santos \\ Universidade Estadual de Campinas
}

August 15, 2018

\begin{abstract}
A finite element formulation is proposed and implemented for analysing the stability of excavated wells using the DiMaggio-Sandler constitutive elastoplastic model with a typical carbonate reservoir configuration. The quality of the finite element approximation is ensured by applying smooth curved elements adapted to the well-bore geometry, and $h-p$ adaptive finite element meshes in the plastic zone. General purpose procedures are defined to transfer the elastoplastic deformation history to newly created integration points. A breakout damage criterion is proposed based on the second invariant of the deviatoric plastic deformation tensor. This damage criterion is used to apply a mesh movement algorithm to represent material collapse. The automatic successive application of the breakout damage criterion results in elliptical realistically looking geometries obtained in experiments reported in the literature.
\end{abstract}

Keywords Geomechanics, Plasticity, Finite elements, Mesh Adaptation, Return-Mapping Algorithm, Welbore stability

\section{Introduction}

During the drilling operation of a well, the in-situ stress state, acquired during millions of years, changes abruptly. Well-bore stability analysis refers to the control of stress changes during the well excavation process, which includes the movement of rock material by the drill bit, and its replacement by the drilling fluid. The confining pressure becomes balanced by the hydrostatic pressure generated by the column of drilling fluids, as illustrated in Figure 1.

The pressure of the drilling fluid is a very important parameter. If the hydrostatic pressure exerted by the column of drilling fluid is excessively high, the fluid may cause the the rock to fracture, causing significant loss of fluid and damage to the reservoir formation (hydraulic fracturing). If the pressure is insufficient, there may be the invasion of the fluid contained in the reservoir. This invasion can cause fluid separation, resulting in expansion of gas contained in the oil (e.g. Deep Horizon disaster). Even when the pressure of the drilling fluid is between the two thresholds mentioned, the stress state change can lead to plastic deformation of the material of the formation and to the eventual collapse of the well. This altered state of stress can be partially controlled by the adjusting the pressure of the drilling fluid by changing its density. 


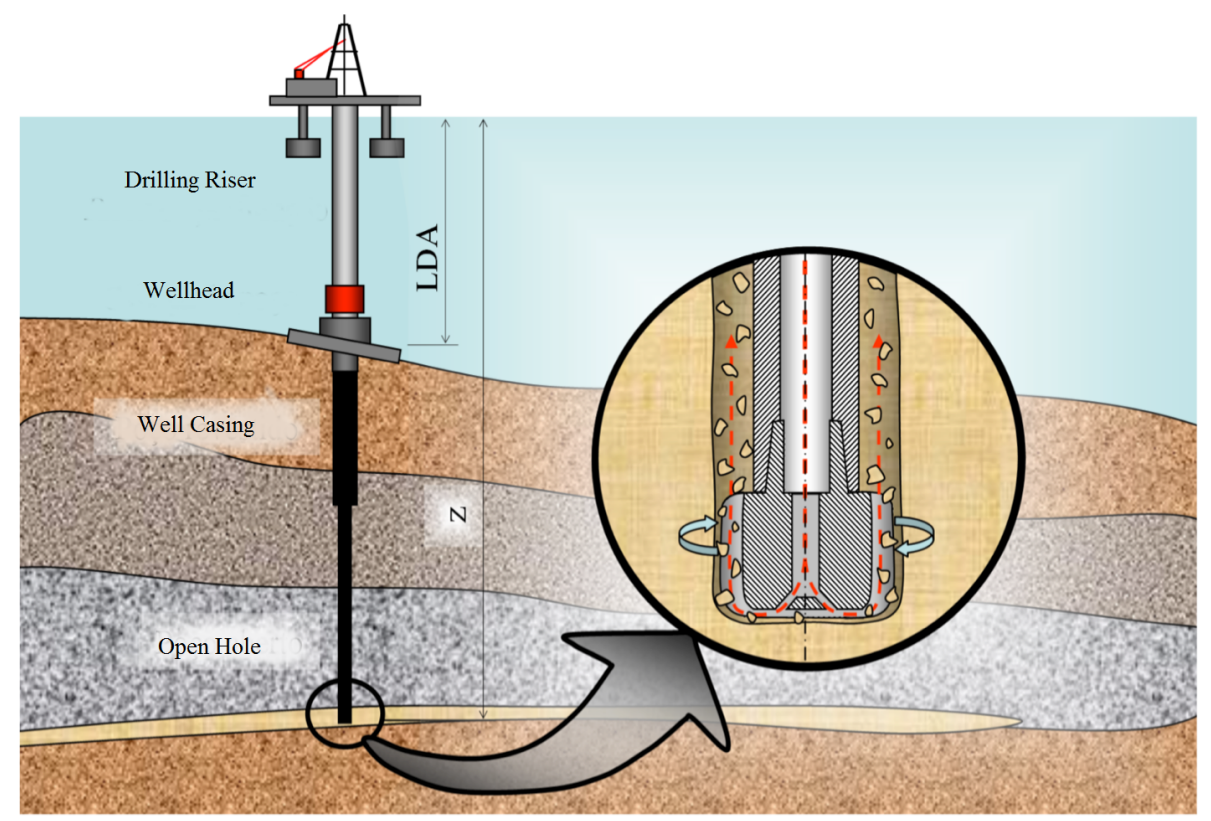

Figure 1: Illustration of an off-shore well drilling process: fluid is pumped and circulated inside the perforation column for lubrication, transport of debrits and stress equilibrium purposes.

Increase of reliability of wells can be obtained by a more detailed study of the stress state around the well, including the non-linear material behavior due to plastic deformation and by including numerical models of breakout of material. In this context, the main purpose of the present article is to study the stress state around the well-bore using computational elastoplasticity [1, 2, 3, 4]. A finite element formulation of the stress state of excavated wells is developed. Additionally, the second invariant of the plastic deformation tensor is adopted as a damage criterion to simulate material collapse (breakout), which is represented numerically by adjusting the geometry of the domain.

The following advanced numerical finite element algorithms have been combined to construct the proposed numerical model of breakout:

- An improved numerical integration algorithm proposed in [5], which holds for associative models.

- $h p$-Refinement around the well-bore. In order to enhance the precision of the elastoplastic simulation, curved meshes directional refined towards the well-bore are used, and the polynomial order is increased in the plastification area.

- A specific transfer procedure for elastoplastic deformation history. Considering the fact that in elastoplastic simulations the stress state is path dependent, adaptive simulations require the communication of the deformation history from one mesh to another.

- An automatic numerical method adapting the geometry of the computational domain by removing the region where the material loss occurs, which is associated to an given value of plastic shear.

Concerning the constitutive plastic model, the focus on this work has been on cases that could test the versatility of the plastic integration algorithm and still resemble the major mechanical response of a rock under high burial stresses. In order to capture the true mechanical behavior of the rock, the shear and traction failure modes must be reproduced. Rocks are materials with intrinsic nonlinear mechanical response. Although all the nonlinearities, such as those in the elastic behavior, cap envelop with hardening/softening and non-associativeness 
of plasticity in the shear envelope could possibly be modeled, it is challenging to devise numerical properties to fulfill the most sophisticated constitutive models. Material availability and test facility capabilities often reduce the amount of real material information at hand when determining material parameters. The real rock behaviour also includes pore collapse and excessive dilatation.

The Dimaggio-Sandler model [6, 7] is an accepted model to represent the behavior of soil materials, and can reproduce all the phenomena described above. Originally, this model has been conceived for a specific sand material, but the perfectly-plastic shear and strain hardening compression cap potentials have shown good overall match of the laboratory observed rock behavior [8, 9]. Based on these facts, the Dimaggio-Sandler model [6, 7] has been chosen to test the versatility of the plastic integration algorithm adopted in the currently proposed numerical finite model.

It could be argued that the use of associative models to simulate the behavior of brittle and dilative materials may be limited. But this behavior happens at low confining pressures. At high confining pressures the cap plasticity is dominant and the material compacts. This is what happens in the reservoir and in the nearwellbore domains, except at the wellbore walls where shear and shear dilation causes breakouts. However, this shear dilation occurs at small hydrostatic stresses and thus small shear strength, presenting a low impact in the overall cavity stresses. However, the "controled dilation" of the Dimaggio-Sandler model, caused by volume expansion, cap softening and transition from shear envelope to cap envelop plasticity, can be used to reduce this effect. These reasons also justify the choice of this model in the present numerical study.

Note also that other models have also been tested in the research that supported this paper. For instance, the Lade-Kin model, in its complete version with the 12 parameters, had been implemented and tested, but it was early abandoned due to its large amount of parameters, lack of clear mechanical meaning of each individual parameter. This mechanical meaning of each parameter is important in the material characterization so that the modelers are prone to detect misleading extrapolation along non-tested load paths. Other important aspect is that this model comprehends two distinct envelopes with a discontinuity in the derivatives in their interception. The code has been devised to support sub-differentiation in a numerical manner and this capability needed to be tested. A more conventional Cam-Clay constitutive model could have been used, but would not test this functionality.

Numerical analyses for well stability and breakout simulations have been previously considered in the literature. For instance, the study in [10] shows how to determine the collapse and fracture-initiation pressure in inclined boreholes using an ideal linear elastic and isotropic model. The conclusion is that the the borehole collapse at low pressures is caused by shear failure in combination with tensile failure. Using von Mises yield condition, the increase in borehole angle does not increase the sensitivity toward collapse. The method presented in [11] determines the failure criteria from limited unconsolidated samples. The simplicity of the approach allows realistic appreciation of the mechanical characteristics derived in laboratory. A conclusion is that unconsolidated formation appears to react mechanically in similar manner to consolidated formation. Well stability analysis for horizontal or highly inclined wells in weak formations is considered in [12], showing that local failure initiation does not necessarily lead to a global borehole instability for some anisotropic rocks. The work in [13] concerns with the development of a shear failure criterion, which is a combination of the effective strength concept and the Drucker-Prager criterion, objecting to quantify the stresses at which borehole breakout will occur. Furthermore, it is shown that the mechanical stability of inclined wells can be improved by adopting optimum deviation angle and drilling direction. The work in [14] presents numerical examples and laboratory tests to show the onset of failure and the growth of the plastic zone development and propagation during production. A method is proposed in [15] for the calculation of linear thermo-poroelastic tangential stresses around a borehole, in a medium with isotropic permeability for the case of constant borehole temperature and pore pressure change. In [16] the authors consider the stability of hollow cylinders with holes of various geometries, which is analyzed experimentally and numerically. They showed that the elliptical breakouts are a more stable structure in terms of hole stability. The numerical simulations showed that the stability of the hole increases with increasing breakouts depth, i.e. the breakout propagation is a stable process where higher 
external stress is required to drive further the breakout. Laboratory tests simulating a breakout in a vertical well are presented in [17] using a synthetic sandstone. The same problem is solved using the commercial finite elements software Ansys and using analytical calculations, with good correlation results.

Adaptive numerical treatment of problems by high order finite element schemes has proved to be very effective [18], by incorporating local mesh refinement ( $h$-adaptivity) and/or different orders of approximating polynomials on separate elements in the same mesh ( $p$-adaptivity). These methods are usually based on a posteriori error control which has been well established for linear problems. For elastoplasticity, and using a representation in terms of variational inequalities, this analysis is more recent [19].

Our adaptive approach is more empirical, and the purpose is to guarantee high quality resolution in the part of the domain where the material is subjected to plastic deformation. A refinement criterion is introduced to define regions where the material has disintegrated, which is based on the magnitude of plastic deformation second invariant. Then, a numerical procedure is constructed to automatically adapt the geometry of the computational domain by removing the region where the values of the refinement indicator is larger than a given threshold.

Implementation of $h p$-adaptive strategies is generally more complex than for standard finite element schemes, since the differences in polynomial orders and the presence of hanging sides introduce additional difficulties in the enforcement of continuity constraints [20]. The mesh refinement process for elastoplastic problems is even more challenging. The tension at an integration point in the domain depends not only on the current deformation state, but also on its loading history. Therefore, a general purpose procedure had to be developed to transfer the elastoplastic deformation history to newly created integration points.

The text is organized in the following way. In Section 2 the elastoplastic mathematical model is described, and its discretized version is summarized in Section 3. The verification of the numerical model is presented in Section 4. The developed method for the breakout simulation is documented in Section 5, and the obtained results are discussed. Some important aspects of the adopted constitutive plastic model are presented in A.

The proposed numerical model has been implemented using the NeoPZ ${ }^{1}$ library [21, 22], which is an object-oriented programming environment providing a framework for the development of finite element simulations. Specific classes have been developed and integrated into NeoPZ for the implementation of elastoplastic constitutive models.

\section{Mathematical Elastoplastic Model}

The mathematical elastoplastic model is composed of two parts: the equilibrium equation coupled with a constitutive initial value problem, as described in [2].

\subsection{Finite Element Formulation for the Mechanical Equilibrium Problem}

Given the reservoir occupying the region $\Omega$, under the action of body force $\vec{b}$, traction $\vec{\tau}$ applied at the boundary part $\Gamma_{N} \subset \partial \Omega$, and assuming no displacement elsewhere at $\Gamma_{D}=\partial \Omega \backslash \Gamma_{N}$, the infinitesimal quasi-static mechanical problem consists in finding the displacement field $\vec{u} \in \mathbf{V}$ such that the weak statement of the equilibrium equation is satisfied

$$
\int_{\Omega} \boldsymbol{\sigma}: \nabla \vec{v} d \omega-\int_{\Omega} \vec{b} \cdot \vec{v} d \omega=\int_{\Gamma_{N}} \vec{\tau} \cdot \vec{v} d s \quad \forall \vec{v} \in \mathbf{V},
$$

where $\mathbf{V}=\left\{\vec{q} \in \mathbf{H}^{1}(\Omega) \mid \vec{q}=0\right.$ on $\left.\Gamma_{D}\right\}, \boldsymbol{\sigma}=\boldsymbol{\sigma}(\varepsilon)$ is the solution of the constitutive initial value problem, as described in the next section, and the deformation tensor is

$$
\varepsilon=\frac{\nabla \vec{u}+\nabla \vec{u}^{T}}{2} .
$$

\footnotetext{
${ }^{1}$ http://github.com/labmec/neopz
} 


\subsection{Constitutive elastoplastic model}

According to [2], an elastic-plastic constitutive model defines the history of an irreversible dissipative process to which the material has been submitted, which is defined by three fundamental axioms: the plasticity criterion, the flow rule, and the hardening law. They are defined in terms of a plasticity function $\Phi$ and a plastic potential function $\Psi$.

The history of the infinitesimal deformation tensor $\varepsilon(t), t \in\left[t_{0}, T\right]$ allows to compute the evolution of the plastic deformation tensor $\varepsilon^{p}(t)$, the internal damage variable $\boldsymbol{\alpha}(t)$ and a plastic multiplier $\dot{\gamma}(t)$ through a system of ordinary differential equations, as described in $\mathrm{A}$

In the present paper, we shall adopt the associative DiMaggio-Sandler plastic model proposed in [6], whose definition is included in A.1. It was conceived for granular soils, and is currently widely applied in the oil industry to represent the behavior of rocks subject to large confinement stresses. As described in [9], this model fits well the plasticity envelopes of a weak carbonate reservoir, which exhibits a consistent shear envelop throughout the triaxial tests under different hydrostatic loadings. It fits well even in those cases where the rock is compacted prior to shear breaking, meaning that the cap plasticity with hardening took place prior to the achievement of a perfectly-plastic shear envelope. More information can found in [9]

\section{Numerical Elastoplastic Model}

The numerical model for the elastoplastic problem uses a $H^{1}$-conforming finite element discretization of the weak statement (1) of the mechanical equilibrium boundary value problem. The computation of the constitutive law associated with each integration point uses an efficient numerical integration method for the initial value problem corresponding to associative plastic models. These two numerical schemes are summarized in the next sections.

\subsection{Numerical Integration of the plastic model}

An algorithm for the integration of general elasto-plastic constitutive initial value problems is summarized in A It is composed of two major steps: the elastic trial step, and the plastic correction step. For the numerical integration of the DiMaggio-Sandler model an improved algorithm is adopted, which has been proposed in [5] for associative models. It is based on the following aspects:

- The elastoplastic model is represented in terms of the principal stresses.

- For the plastic correction step, a distance function is defined, which has to be minimized in order to determine the projection of the trial stress onto the yield surface (closest point projection). This projection is made possible thanks to the Haigh-Westergaard representation. This geometric interpretation of the plastic corrector step not only improves the numerical efficiency, but also the stability of the computations.

\subsection{Finite Element Model for the Mechanical Equilibrium Problem}

The finite element discretization of the equilibrium problem starts by the construction of a partition $\mathcal{T}=\{K\}$ of the computational domain, and of a finite dimensional approximation space $\mathbf{V}_{\mathcal{T}} \subset \mathbf{V}$, formed by functions which are piecewise defined in terms of polynomials based on $\mathcal{T}$. Then, the approximate solution $\vec{u}_{\mathcal{T}} \in \mathcal{T}$ is searched such that the variational formulation (1) is verified for all $\vec{v}_{\mathcal{T}} \in \mathbf{V}$.

Due to the vertical extension of the problem, we shall consider a two dimensional plane strain model, and restrict the computational region $\Omega$ to one quadrant. The computational domain representing the reservoir, the boundary conditions, and the geometrical mesh are illustrated in Figure 2 . Because of the symmetry properties, 


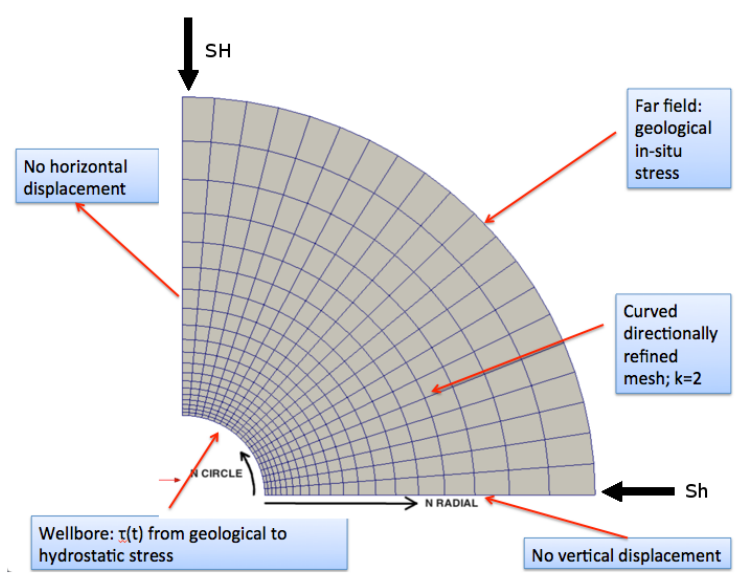

Figure 2: Computation domain and boundary conditions.

no penetration is imposed at $\Gamma_{D}=\{(x, 0),(0, y) \mid 0<x, y<1\}$. At the well-bore, the normal stress corresponds to the hydrostatic stress of the drilling fluid, and at the far field the normal stress is equal to the in-situ stress. In our simulation the gravity effects are neglected, so that $\vec{b}=0$.

The stress state is sensitive to the accuracy of geometry representation. Element removing and/or non smooth boundary produce concentration of non physical stresses. The use of smooth curved elements adapted to the well-bore geometry is crucial. Thus, in order to enhance the precision of the elastoplastic simulation, elements with quadratic geometry are used, and they are directional refined towards the well-bore, as shown in Figure 2

Constant polynomial degree $k=2$ is adopted for approximations in the absence of material damage. However, for simulations involving breakout, the elements that intersect the plastic region are divided and their polynomial orders are increased, as described in Section 5. The construction of shape functions and assembly of the adopted $H^{1}$-conforming finite element approximation spaces are described in [23, 24, 20] for $h, p$ and $h p$-adaptive curved meshes.

\section{Verification Tests}

In order to verify the implementation of the proposed finite element numerical model, we simulate the stress state around a well-bore and compare the computed solutions with the analytic Airy solution for the elastic stress state [25]. The stress state is given by the DiMaggio-Sandler model, whose parameters are defined in Table 1. corresponding to a carbonate reservoir material of the Brazil Offshore Santos Basin. Its characterization has been presented in paper [9]. The well radius is $r_{w b}=0.10795 \mathrm{~m}$, the reservoir radius is $r_{e}=3 \mathrm{~m}$, and the effective in situ tension components are maximum horizontal stress in the $y$-direction $S_{H}^{\prime}=-62.1 \mathrm{MPa}$, minimum horizontal stress in the $x$-direction $S_{h}^{\prime}=-45.9 \mathrm{MPa}$, and vertical stress $S_{v}^{\prime}=-48.2 \mathrm{MPa}$.

The mesh configuration for this test is obtained by 40 subdivisions in the radial direction, and 80 circular ones, and the polynomial degree is $k=2$. The results are documented in Figure 3 by the graphs of the values of the plasticity function $\Phi$ along the well-bore circular curve. Three cases are represented, corresponding to different values of well-bore fluid pressures $P_{f}^{\prime}$. The corresponding values of the yield function $\Phi\left(I_{1}, \sqrt{J_{2}}, L\right)$, the plastic deformation second invariant $\sqrt{J_{2}\left(\varepsilon^{p}\right)}$, the first invariant of the stress tensor $I_{1}(\boldsymbol{\sigma})$ and the second invariant of the deviatoric stress tensor $J_{2}(\boldsymbol{\sigma})$ are graphically represented in Figure 4 Figure 6 


\begin{tabular}{|c|c|}
\hline Parameter & Value \\
\hline \hline$E$ (elasticity modulus) & $29269 \mathrm{MPa}$ \\
\hline$\nu$ (Poisson coefficient) & 0.203 \\
\hline$A$ & $152.54 \mathrm{MPa}$ \\
\hline$B$ & $0.0015489 \mathrm{MPa}^{-1}$ \\
\hline$C$ & $146.29 \mathrm{MPa}$ \\
\hline$D$ & $0.018768 \mathrm{MPa}^{-1}$ \\
\hline$R$ & 0.91969 \\
\hline$W$ & 0.06605 \\
\hline
\end{tabular}

Table 1: Parameters for DiMaggio-Sandler elastoplastic model.

Case 1: $P_{f}^{\prime}=28.9 \mathrm{MPa}$ In Figure 3 (top left side), the plot for the yield function along the well-bore circular curve is such that $\Phi<0$, which means that the problem is purely elastic. The values of $\Phi\left(I_{1}, \sqrt{J_{2}}, L\right)$, $\sqrt{J_{2}\left(\varepsilon^{p}\right)}, I_{1}(\boldsymbol{\sigma})$ and $J_{2}(\boldsymbol{\sigma})$ are graphically represented in Figure 4 . As expected, $\Phi<0$ and $\sqrt{J_{2}\left(\epsilon^{p}\right)}=0$.

Case 2: $P_{f}^{\prime}=23.4 \mathrm{MPa}$ The results for this case are graphically represented in Figure 5. The lower fluid pressure causes the occurrence of a region with plastic deformation around the well-bore, where $\Phi=0$. The extent of the region corresponds to approximately $1.3 \%$ of the area of the reservoir model. The comparison with the analytic Airy solution in Figure 3 (top right side) shows that the plastification extends to an angle of $46.5^{\circ}$.

Case 3: $P_{f}^{\prime}=19.5 \mathrm{MPa}$ Decreasing the fluid pressure even more, the region with plastific deformation grows, as shown in Figure 6, occupying around $4.28 \%$ of the modeled reservoir area, and extending to a $72.12^{\circ}$ angle at the well-bore curve, as illustrated in Figure 3 (bottom side). As suggested by [26], a safe threshold is $60^{\circ}$. Thus, according to this criterion, the stress state of Case 3 corresponds to a dangerous scenario for well stability. 
Case 1

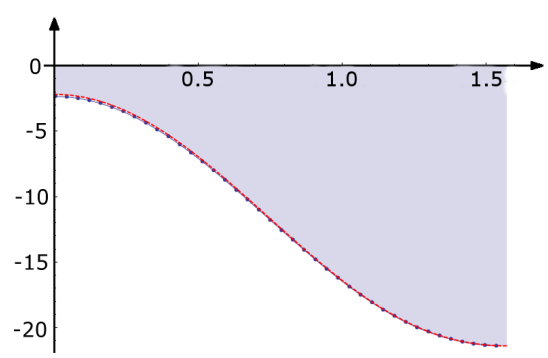

Case 2

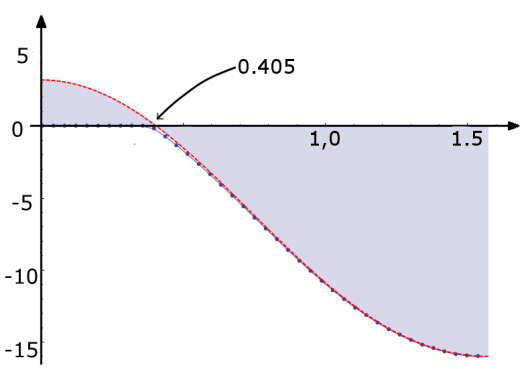

Case 3

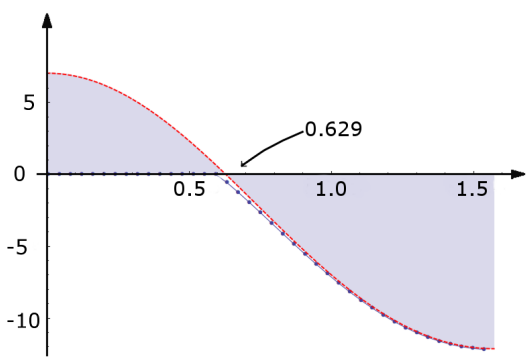

Figure 3: Plasticity function $\Phi(M P a)$ computed at the circular well-bore curve by the Airy solution (red lines) and by the numerical finite element solution (dotted lines) for the Case 1, Case 2, and Case 3.

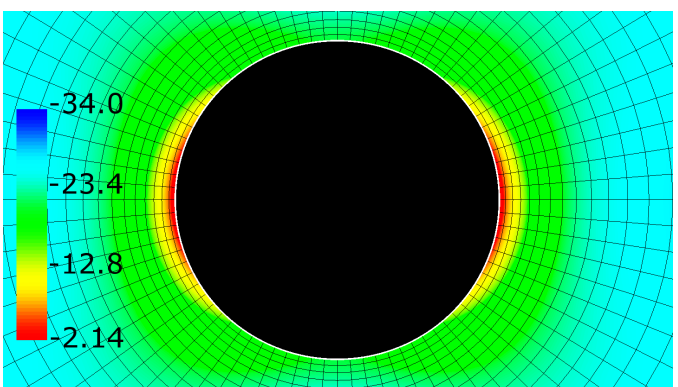

(a) $\Phi\left(I_{1}, \sqrt{J_{2}}, L\right)(M P a)$

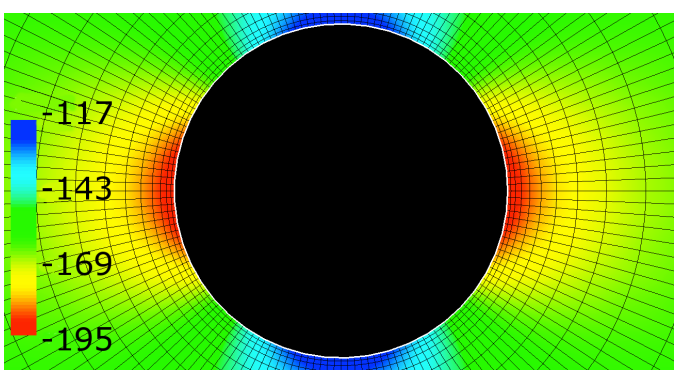

(c) $I_{1}(\boldsymbol{\sigma})\left(M P a^{2}\right)$

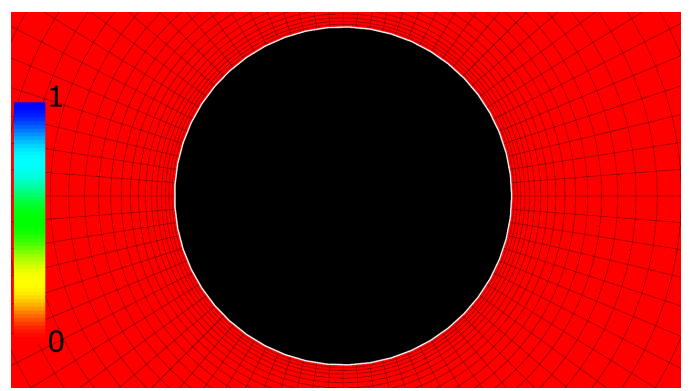

(b) $\sqrt{J_{2}\left(\varepsilon^{p}\right)}$

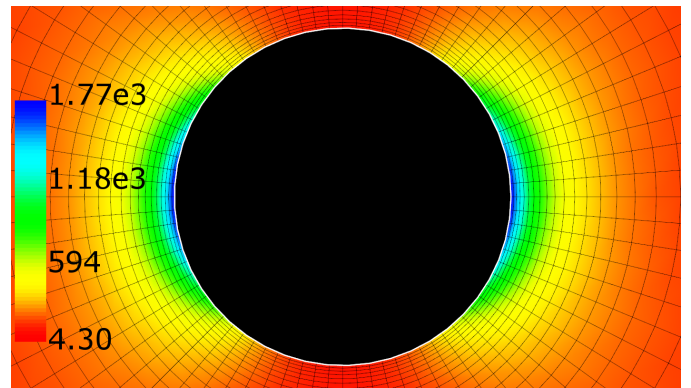

(d) $J_{2}(\boldsymbol{\sigma})(M P a)$

Figure 4: Case 1: $\Phi\left(I_{1}, \sqrt{J_{2}}, L\right)$, and $\left.\sqrt{J_{2}\left(\varepsilon^{p}\right.}\right)$ (top side); $I_{1}(\boldsymbol{\sigma})$, and $J_{2}(\boldsymbol{\sigma})$ (bottom side). 


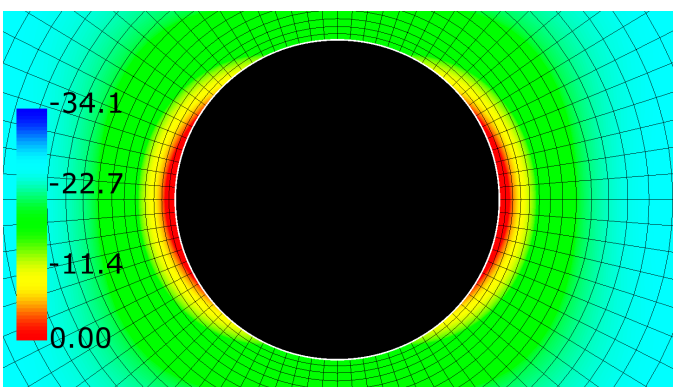

(a) $\Phi\left(I_{1}, \sqrt{J_{2}}, L\right)(M P a)$

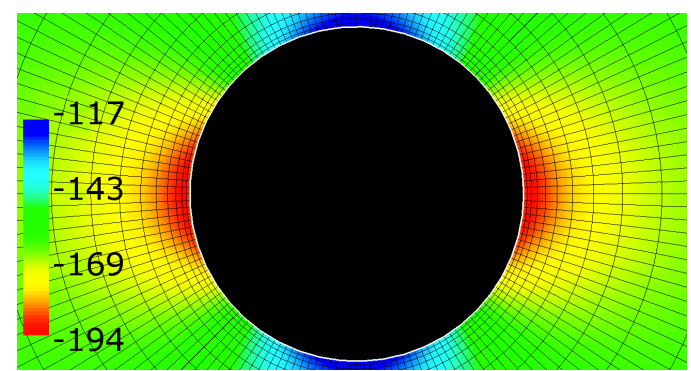

(c) $I_{1}(\boldsymbol{\sigma})(M P a)$

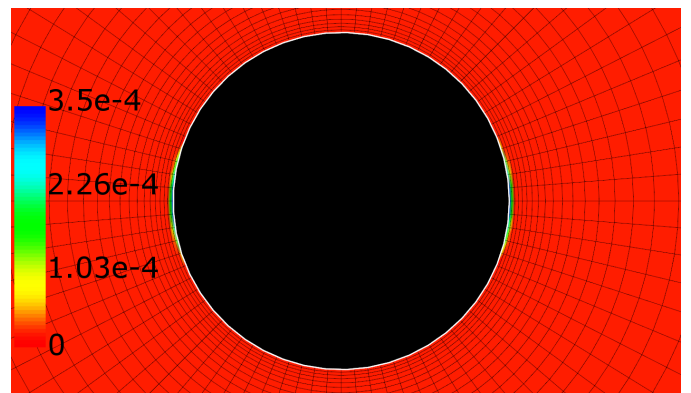

(b) $\sqrt{J_{2}\left(\varepsilon^{p}\right)}$

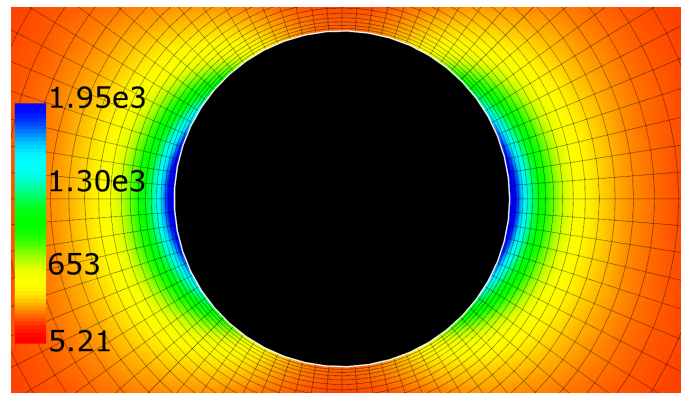

(d) $J_{2}(\boldsymbol{\sigma})\left(M P a^{2}\right)$

Figure 5: Case 2: $\Phi\left(I_{1}, \sqrt{J_{2}}, L\right)$, and $\left.\sqrt{J_{2}\left(\varepsilon^{p}\right.}\right)$ (top side); $I_{1}(\boldsymbol{\sigma})$, and $J_{2}(\boldsymbol{\sigma})$ (bottom side).

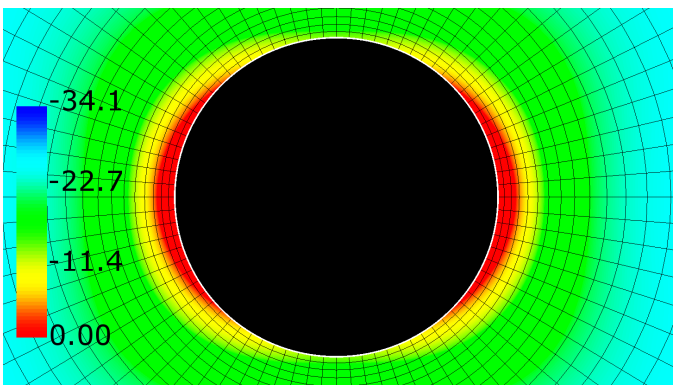

(a) $\Phi\left(I_{1}, \sqrt{J_{2}}, L\right)(M P a)$

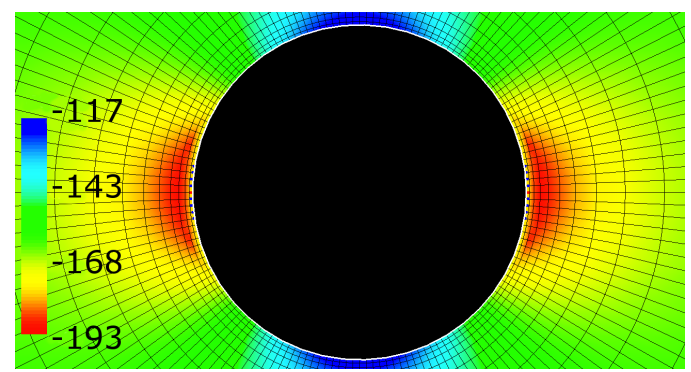

(c) $I_{1}(\boldsymbol{\sigma})(M P a)$

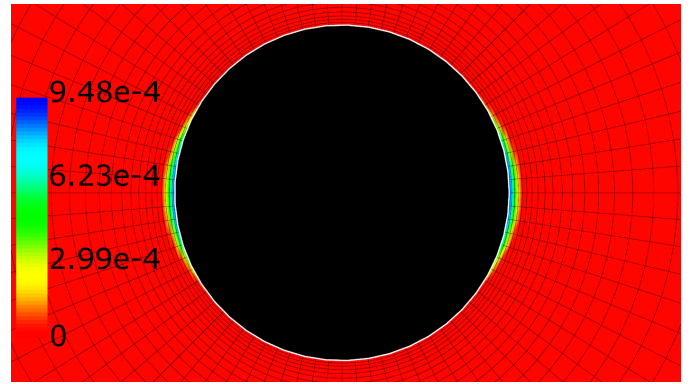

(b) $\sqrt{J_{2}\left(\varepsilon^{p}\right)}$

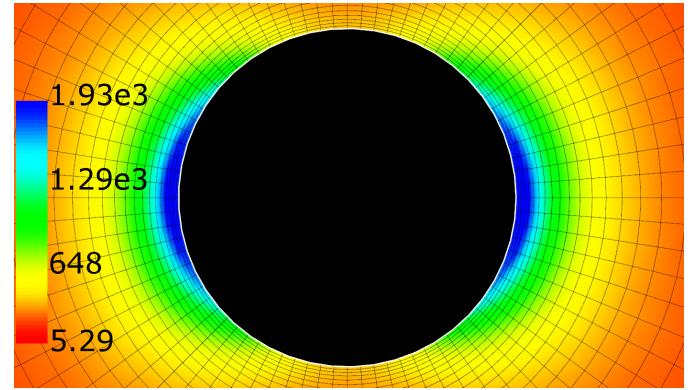

(d) $J_{2}(\boldsymbol{\sigma})\left(M P a^{2}\right)$

Figure 6: Case 3: $\Phi\left(I_{1}, \sqrt{J_{2}}, L\right)$, and $\sqrt{J_{2}\left(\varepsilon^{p}\right)}$ (top side); $I_{1}(\boldsymbol{\sigma})$, and $J_{2}(\boldsymbol{\sigma})$ (bottom side). 
In order to evaluate the effect of discretization resolutions on the numerical solution, the method is applied to the reservoir configuration of Case 3 using three different mesh refinements, and polynomial degrees $k=1,2$ and 3. The first mesh is obtained by 20 subdivisions in the radial direction, and 40 circular ones (Mesh 1). The second (Mesh 2) and third (Mesh 3) finer meshes consists of subsequent uniform subdivisions of the first one. Defining the plasticized region where $\sqrt{J_{2}\left(\varepsilon^{p}\right)}>0$, its area for different discretizations is represented graphically in Figure 8. Figure 7 displays the value of $\sqrt{J_{2}\left(\epsilon^{p}\right)}$ for the solutions on Mesh 2 and Mesh 3, with $k=2$. It can be observed that the plastified area converges as the mesh is refined either in $\mathrm{h}$ or in polynomial degree.

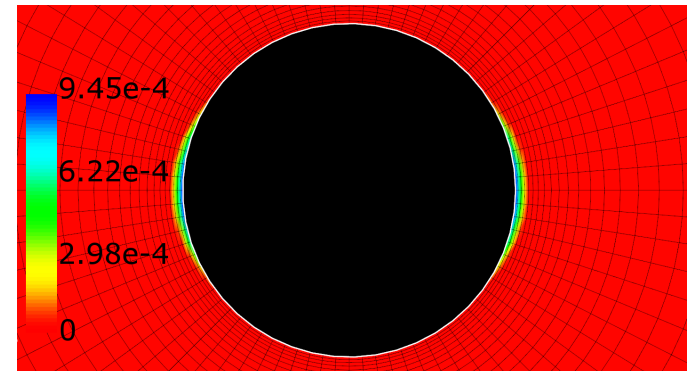

(a) $40 \times 80$ elements: plasticized area $=1.12 \times$ $10^{-3} \mathrm{~m}^{2}$

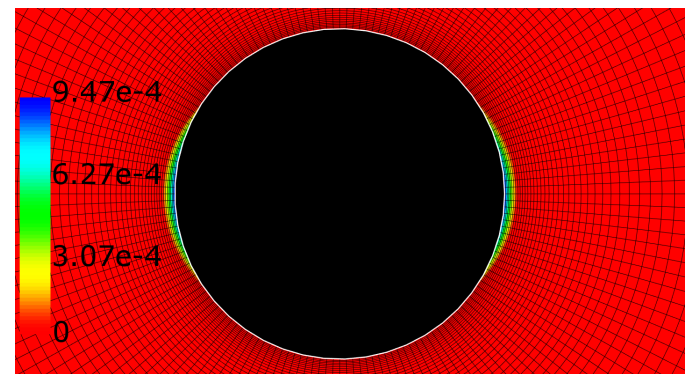

(b) $80 \times 160$ elements: plasticized area $=1.13 \times$ $10^{-3} \mathrm{~m}^{2}$

Figure 7: Value of $\sqrt{J_{2}\left(\epsilon^{p}\right)}$ associated to the solutions obtained with two mesh resolutions and $k=2$.

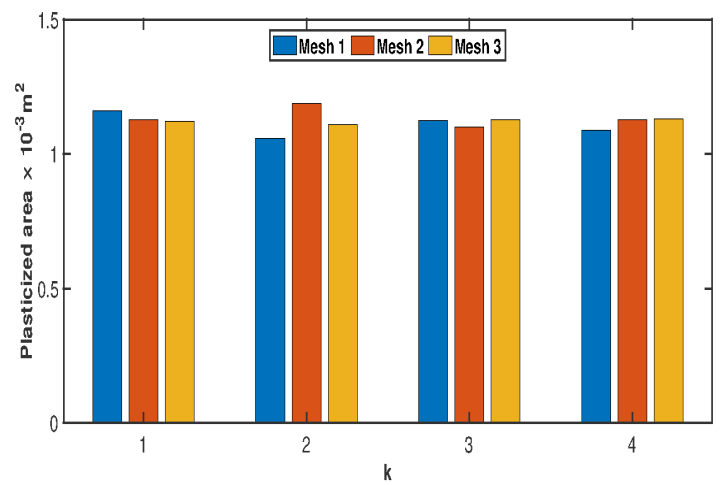

Figure 8: The effect of the mesh resolution and polynomial degree on the plasticized area.

\section{Breakout Simulation}

The breakout phenomenon is characterized by the well-bore mechanical failure and loss of material in the regions where the shear strains are critical. The elastoplastic simulation allows to identify the regions where this is most likely to happen.

In the well drilling area of oil and gas industry, the information of damage extension is at a premium. Well design and stability monitoring while drilling hence rely on generally poor, interpolated information, mainly obtained from correlation from welllogs. On the other hand, rock is heterogeneous, brittle, anisotropic, sometimes is load-rate-dependent, etc. There must be a trade off between model sophistication and applicability. This is the main reason for requiring models based on "mechanically meaningful" parameters, so that correlation from physical properties inferred from the wellogs may be devised, as well as any misjudgements may be 
detected at early stages (parameter-definition stages). Having that in mind, it should become clear that the numerical solution on that backgroung must not be taken literally into consideration. The plastified zone does not necessarily represent a damaged zone which remains attached to the formation, nor continuously. There is insufficient amount of information to deploy real damage models that could determine the volume of fragmented rock debris. There is also insufficient information to state whether the excavation could still be geometrically stable.

The plastified zone or opening angle should be regarded as imperfect quantitative indications of a mechanical damage zone. From the drilling experience, global excavation instability occurs rarely and is always preceded by local wall instabilities (breakout). The formation of breakouts poses more problems to the well cleaning than to the cavity stability, as the breakouts may occur over a large extent along the wellbore axis. The volume of rock debris produced by breakout may easily surpass the amount of cuttings from the drillbit. Pressure fluctuations in the excavation associated to the drillstring vibration ease the freeing of damaged material. The settlement of large amounts of debris around the drillstring may cause it to get stuck and prevent further drilling with some other serious consequences.

Thus, each drilling company rely on ultimate plastified area or on opening angle indications to drive their wellbore stability analysis. However, they usually rely on the simple Mohr-Coulomb shear-envelope-basedplasticity models to evaluate those indicators. Although it captures the shearing processes at the wellbore wall, they are unable to reproduce the amount of compaction experienced by the rock mass under depletion that drives the evolution of far-field stresses.

Our proposal is to use the plastic deformation second invariant $\sqrt{J_{2}\left(\varepsilon^{p}\right)}$ to define a criterion for the regions where the material has disintegrated. As described in the next sections, the numerical method automatically adapts the geometry of the computational domain by removing the region where the values of $\sqrt{J_{2}\left(\varepsilon^{p}\right)}$ are larger than a given threshold. Then, a moving-mesh algorithm is adopted to simulate how the cavity failure is supposed to evolve based on the complete detachment of the plastified zone material.

\subsection{Automatic Procedure for Adjusting the Geometry of the Domain}

An attempt to adapt the geometry simply removing elements with excessive plastic deformation is not a viable solution: the removal of the elements creates nonphysical re-entrant corners causing high stress concentrations that are non physical.

A different approach is considered based on the hypothesis that the isolines of the proposed breakout criterion can be closely matched by ellipses whose center is the well-bore center. This same geometry can also be observed in experiments conducted by [26]. Based on this principle, a numerical method has been developed [25] to adjust the axes of an ellipse to points with constant values of the breakout criterion $\sqrt{J_{2}\left(\varepsilon^{p}\right)}$.

A motivation for this choice of criterion is that the plastic deformation is characterized by two components, one hydrostatic and the other deviatoric. One can conjecture that the hydrostatic deformation does cause the material to fail. The shear deformation, on the other hand, causes the sliding between the microscopic plains possibly leading to rupture. Hence the value of $\sqrt{J_{2}\left(\varepsilon^{p}\right)}$ has been chosen as a criterion to quantify the tendency of the material to disintegrate.

The computation of the axes of the ellipse corresponding to a given value of $\sqrt{J_{2}\left(\varepsilon^{p}\right)}$ is performed using a standard least square algorithm. Precisely, a list with coordinates $\left(x_{i}, y_{i}\right)$ of points with constant value of $\sqrt{J_{2}\left(\varepsilon^{p}\right)}$ are computed. The error function $E(a, b)=\sum_{i}\left(x_{i}-a \sqrt{1-\frac{y_{i}^{2}}{b^{2}}}\right)$ is defined to measure the deviation of the points $\left(x_{i}, y_{i}\right)$ from a general ellipse with focus at $(0,0)$. Then, the least squares method is used for the determination of the values $a$ and $b$ minimizing this error function.

Once the ellipse equation is found, the mesh nodes on the contour of the well-bore are moved to fit the position of the ellipse. The remaining nodes of the mesh that are moved to maintain the smoothness of the mesh. 


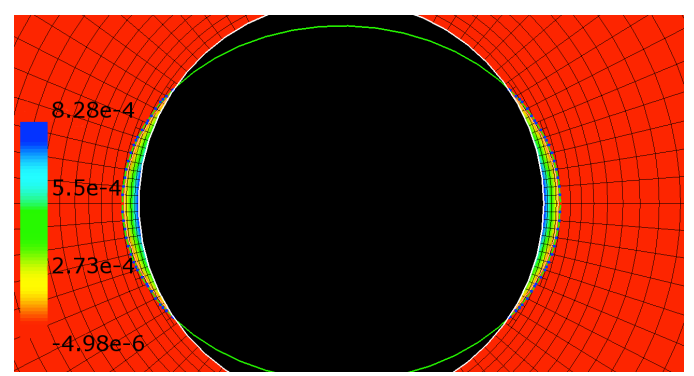

Figure 9: Boundary adjustment using an ellipse shape.

\subsection{Refinement Process on the Plastic Zone}

The mesh refinement process for elastoplastic problems is challenging. The tension at an integration point in the domain depends not only on the current deformation state, but also on its loading history. For meshes without adaptation, this history can be obtained by storing the history of the nodal displacements. In the case of mesh movement, the displacements themselves do not allow to reconstruct the history of deformation. During the refinement process, integration points are created and others are deleted. The adopted technique to refine meshes arbitrarily is to implement a general procedure that allows to compute the solution at an arbitrary point in a mesh. At each refinement step a new mesh object is created and the deformation history is obtained by computing the history of deformation at a given coordinate by computing the incremental deformation at all previous meshes (e.g. solutions). For more details see [25]. The implementation has two fundamental steps:

1. Identify the element and its coordinates in the master element domain corresponding to an $(x, y)$ coordinate.

2. Submit the newly created material point to the deformation tensor evolution according to the solution of the previous meshes.

Refinements $h$ and $p$ in the plasticized zone are illustrated in Figure 10.

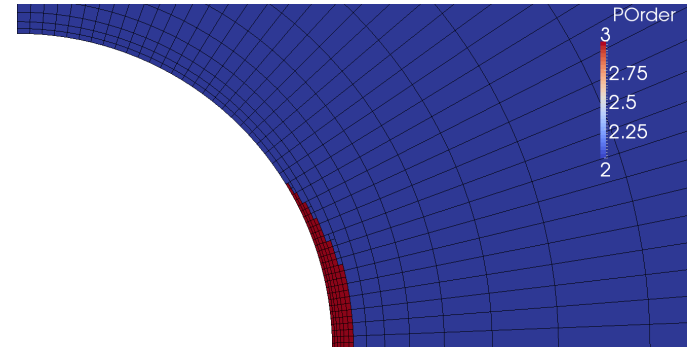

(a) $p$-refinement.

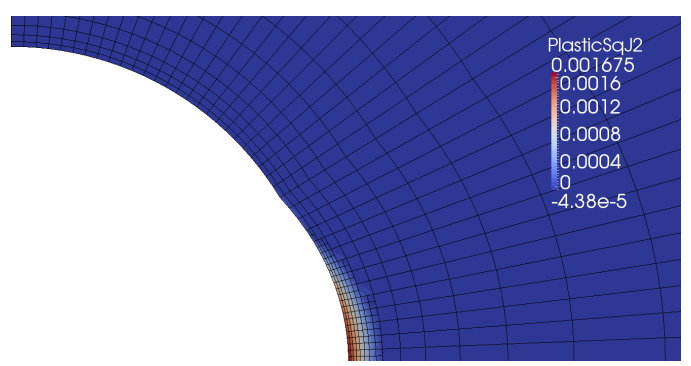

(b) $h$-refinement

Figure 10: Illustration of $h$ and $p$ mesh refinements on the plasticized zone.

\subsection{Results}

The geometry adjustment and the $h p$ mesh refinement are illustrate by numerical results for the confinement state configuration in Case 3, described in Section 4. This choice is justified because, among the three considered cases, this is the worst case scenario, since the breakout damage has a breakout angle greater than $60^{\circ}$, as detailed in the reference [26]. Two parameters are defined: RefHP and $A d j B K$. In a first step, elements that 
have $\sqrt{J_{2}\left(\varepsilon^{p}\right)}>\operatorname{RefHP}$ are searched in the mesh, were an $h p$ mesh refinement is performed. Secondly a geometric adjustment criteria is specified to search a line with constant values of $\sqrt{J_{2}\left(\varepsilon^{p}\right)}=A d j B K$, which is adjusted by an ellipse. Therefore, four geometry adjustment stages are performed. Precisely,

1. Given an initial well-bore geometry, the problem is initially solved with $p=2$, as described in Section 4 .

2. A mesh adjustment procedure is performed, subdividing each element that has $\sqrt{J_{2}\left(\varepsilon^{p}\right)} \geq \operatorname{RefHP}$, and increasing it's polynomial order to $p=3$.

3. The geometry is adjusted based on the criterion $A d j B K$ and the problem is solved in the original mesh that is restricted to the new geometry.

\section{Return to step 1.}

The results in Figure 11 are for an initial mesh with $40 \times 80$ elements (Test 1$)$. The values for $\sqrt{J_{2}\left(\varepsilon^{p}\right)}$ are shown at four instances of the breakout simulation. The thresholds $A d j B K=\operatorname{RefHP}=0.0001$ were chosen such that the relevant parts of the domain where plastification occurs are refined. For comparison, the curves corresponding to $\sqrt{J_{2}\left(\varepsilon^{p}\right)}=0.0001$ obtained at the four stages are presented in all figures, and plotted in white color. At the first instance, shown in Figure 11 (a), the results are for the original geometry region, and the $h p$ adapted mesh is shown. At this initial instant, a maximum second invariant plastic deformation value $\sqrt{J_{2}\left(\varepsilon^{p}\right)}=0.000829$ is observed. In a next simulation instant, represented by Figure 11 (b), the geometry adjustment is performed, by the removal of the plasticized area delimited by the ellipse that has been adjusted to the points where $\sqrt{J_{2}\left(\varepsilon^{p}\right)}=\operatorname{Adj} B K$ (first white isoline seen in Figure 11 (a)). At this instant, a maximum value $\sqrt{J_{2}\left(\varepsilon^{p}\right)}=0.001669$ is observed. Again, a white isoline is used to mark the points where $\sqrt{J_{2}\left(\varepsilon^{p}\right)}=A d j B K$. Next, the plasticized region delimited by this isoline is removed and the simulation returns to stage 1. Figure 11 (c) indicates the next result, after the $h p$ refinement and running the problem in a new mesh. It is observed a maximum value of $\sqrt{J_{2}\left(\varepsilon^{p}\right)}=0.003159$, which gives an evidence for a plastic deviatoric deformation increasingly higher. Figure 11 (d) shows the result of the last procedure instance. The last maximum value $\sqrt{J_{2}\left(\varepsilon^{p}\right)}=0.006338$ is more than twice the value observed in the previous step and the plasticized area is considerably reduced.

Two tendencies have been noted. One is the tendency of the plasticized zone to move towards the inferior symmetry axis, with decreasing angle of plastic deformation extent. The other evidence is that the plasticized area decreases. The data shown in Table 2 for removed and plasticized areas, damage extension and breakout opening angles at the four breakout simulation instants described before confirm these tendencies. Note that in well-bore projects the angle of plastic deformation extent is an important parameter, and it is usually computed based on linear elastic stress states, and limited to a predefined value.

\begin{tabular}{|c|c|c|c|c|c|c|c|c|c|}
\hline \multirow{2}{*}{ Step } & \multicolumn{3}{|c|}{ removed area $\left(\times 10^{-3} \mathrm{~m}^{2}\right)$} & \multicolumn{3}{|c|}{ plasticized area $\left(\times 10^{-3} \mathrm{~m}^{2}\right)$} & \multicolumn{3}{|c|}{ damage area $\left(\times 10^{-3} \mathrm{~m}^{2}\right)$} \\
\cline { 2 - 11 } & Test 1 & Test 2 & Test 3 & Test 1 & Test 2 & Test 3 & Test 1 & Test 2 & Test 3 \\
\hline 1 & - & - & - & 1.148 & 1.113 & 1.126 & 1.148 & 1.113 & 1.126 \\
\hline 2 & 1.173 & 1.122 & 1.124 & 1.238 & 1.235 & 1.222 & 2.411 & 2.357 & 2.346 \\
\hline 3 & 2.441 & 2.379 & 2.386 & 1.203 & 1.181 & 1.221 & 3.644 & 3.560 & 3.607 \\
\hline 4 & 3.688 & 3.615 & 3.719 & 1.010 & 1.069 & 1.168 & 4.698 & 4.684 & 4.887 \\
\hline
\end{tabular}

Table 2: Breakout evolution using different mesh configurations: with $h p$ adapted meshes with initial uniform partition of $40 x 80$ and polynomial order $p=2$ (Test 1) and $80 x 160$ elements, and polynomial degree $p=2$ (Test 2), and without adaptativity, using the fine mesh of $80 \times 160$ elements and polynomial degree $p=3$ (Test $3)$.

In Table 2 simulation results are presented for two other discretization configurations. Test 1 refers to an adaptive simulation starting from an initial uniform mesh with $40 \times 80$ elements and $k=2$. Test 2 refers to an 


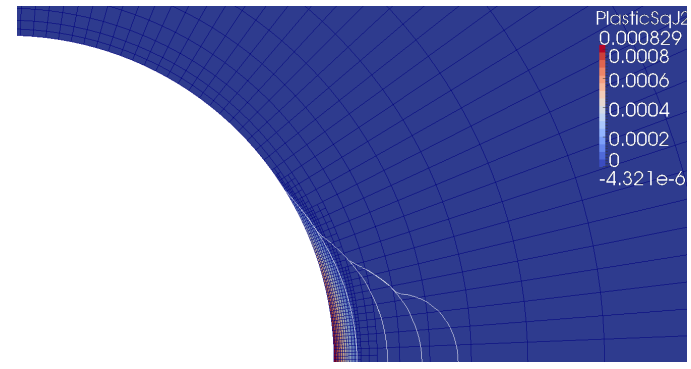

(a) Step 1

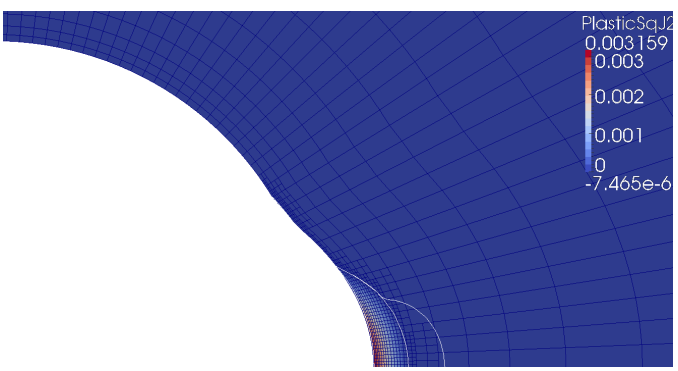

(c) Step 3

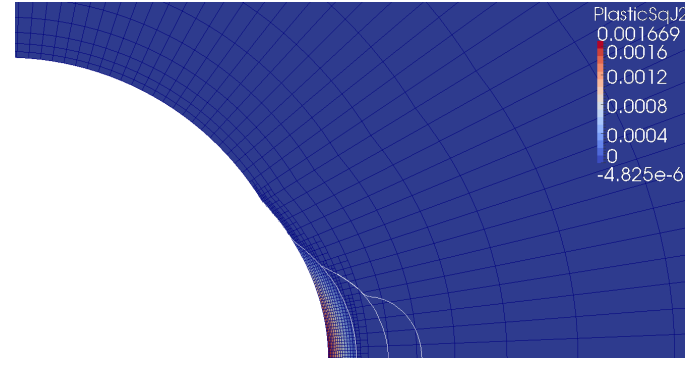

(b) Step 2

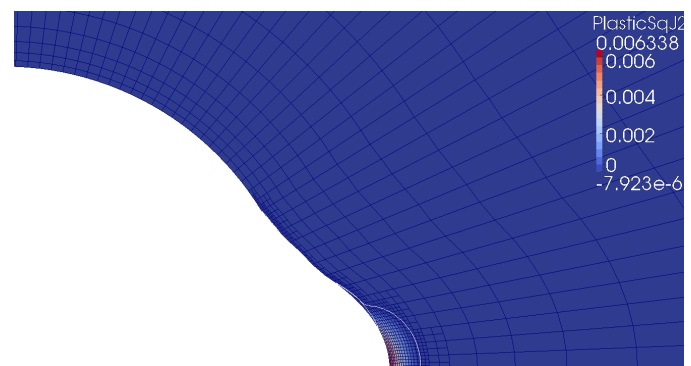

(d) Step 4

Figure 11: Breakout simulation: automatic geometric fitting to the plastic zone.

adaptive simulation starting from a refined initial uniform mesh, with $80 \times 160$ elements and $k=2$, and Test 3 uses a mesh with $80 \times 160$ elements in all stages and $k=3$, without applying further $h p$ adaptive strategy. The results of the removed, plasticized and damage areas are in close agreement in all the three strategies. This table shows that the extent of plastic deformation and/or breakout is stable with respect to the choice of initial mesh and polynomial order of approximation.

\section{Conclusions}

The DiMaggio-Sandler elastoplastic model has been applied to the study of well-bore stability, applying hpadaptive finite element approximations combined with mesh movement. The robustness of this elastoplastic model is greatly improved by parametrizing the plastic yield surface and performing proper tensor analysis. A failure criterion is proposed assuming that the material will disintegrate due to excessive shear strain. Based on this criterion, the quality of the finite element simulation is guaranteed by applying $h$ and $p$ refinements in the region were plastic deformation exceeds a given old. For the newly created adaptive meshes, the complete deformation history is applied to each integration point. Stacks of meshes are used to keep track of this deformation history. After this adaptive simulation, an automatic elliptic geometry fitting is applied to the region of excessive deformation, and the corresponding material is removed, by using mesh movement. One of the results of our work is that using the automatic successive application of the breakout damage criterion, and after removing the area with excessive plastic deformation, realistically looking geometries are obtained, as compared with experimental results reported in the literature, the area of plastic deformation migrating towards the horizontal line, with decreasing angle of plastic deformation extent. 


\section{Acknowledgments}

The authors thankfully acknowledges financial support from ANP-Brazilian National Agency of Petroleum, Natural Gas and Biofuels (grant SAP4600333146). P. R. B. Devloo and S. M. Gomes also thank financial support from CNPq - the Brazilian Research Council ((305425/2013-7, and 304029/2013-0, respectively). The authors also thank O. Durán, T. Quinelato, and L. Marques for the help in setting some NeoPZ configurations for the current simulations.

\section{References}

[1] Chen WF, Han DJ. Plasticity for Structural Engineers. J. Ross Publishing; 2007. 310 p.

[2] Neto EAS, Peric D, Owen DRJ. Computational Methods for Plasticity. Wiley; 2009. 791 p.

[3] Borja RI. Plasticity: Modeling \& Computation. Springer-Verlag Berlin Heidelberg; 2013. 255 p.

[4] Lubliner J. Plasticity theory. Dover Publications; 2008. 540 p.

[5] Cecílio DL, Devloo PRB, Gomes SM, Santos ESR and Shauer N. An improved numerical integration algorithm for elastoplastic constitutive equations. Computers and Geotechnics 2015;64:1-9.

[6] DiMaggio FL, Sandler I. Material Model for Granular Soils. Engineering Mechanics Division 1971;97:935-950.

[7] Sandler IS, Rubin D. An algorithm and a modular subroutine for the cap model. Int J Numer Anal Methods Geomech 1979;3:173-186.

[8] Santos ESR. Simulador de meios porosos saturados elastoplásticos. Universidade Estadual de Campinas. 2009.

[9] Santos ESR, Ferreira FH. Mechanical Behavior of a Brazilian Off-Shore Carbonate Reservoir. US Rock Mechanics Symposium - ARMA 2010.

[10] Aadnoy BS, Chenevert ME. Stability of Highly Inclined Boreholes. SPE Drilling Engineering 1987; SPE 16052.

[11] Somerville JM, Smart BGD. The Prediction of Well Stability Using the Yield Zone Concept. SPE Drilling Engineering 1991; SPE 23127.

[12] Morita N, Ross CK. Core-Based Horizontal or Highly Inclined Well Stability Analysis for Unconsolidated Formations. SPE Drilling Engineering 1993; SPE 26332.

[13] Shaohua Z, Hillis RR, Sandiford M. On the Mechanical Stability of Inclinate Wellbores. SPE Drilling and Completion 1996; SPE 28176.

[14] Morales RH, Webb TR, Hollier R. Borehole Failure: Safe Drawdown Pressures and Wellbore Damage Radius. SPE Drilling Engineering 2000; SPE 58789.

[15] Wanga Y, Dusseault MB. A coupled conductive-convective thermo-poroelastic solution and implications for wellbore stability. Journal of Petroleum Science and Engineering 2003; 38:187-198

[16] Papamichos E, Liolios P, Hoek PJ. Breakout stability experiments and analysis. American Rock Mechanical Association 2004 
[17] Villarroel FMG, Junior EVA, Rabello GF, Block M, Azevedo VA. Breakouts: Physical and Numerical Models. SPE Drilling Engineering 2010; SPE 131656.

[18] Ainsworth M, Oden J. A posteriori error estimation in finite element analysis. Wiley-Interscience Series of Texts, Monographs, and Tracts. Wiley \& Sons, Inc., New York. 2000.

[19] Carstensen C, Schröder A and Wiedemann S. An optimal adaptive finite element method for elastoplasticity. Numer Math 2016;132:131-154.

[20] Calle JLD, Devloo PRB, Gomes SM. Implementation of continuous h p -adaptive finite element spaces without limitations on hanging sides and distribution of approximation orders. Comput Math Appl 2015;70:1051-1069.

[21] Devloo PRB. PZ: An object oriented environment for scientific programming. Comput Methods Appl Mech Eng 1997;150:133-153.

[22] Devloo PRB. Object Oriented Tools for Scientific Computing. Eng Comput 2000;46:203-214.

[23] Devloo PRB, Bravo CMA, Rylo EC. Systematic and generic construction of shape functions for padaptive meshes of multidimensional finite elements. Eng Comput 2009;198:1716-1725.

[24] Lucci PCA. Descrição Matemática de Geometrias Curvas por Interpolação Transfinita. Universidade Estadual de Campinas. 2009.

[25] Cecílio DL. Modelagem Elastoplástica Aplicada à Simulação Numérica da Estabilidade de Poços de Petróleo. Universidade Estadual de Campinas. 2014.

[26] Zoback MD, Moos D, Mastin L. Wellbore breakouts and in situ stress. Journal of Geophys Res 1985;90:5523-5530.

\section{A Constitutive elastoplastic model}

As studied in [2], the total deformation tensor $\varepsilon$ is divided into two components: $\varepsilon=\varepsilon^{e}+\varepsilon^{p}$, an elastic strain $\varepsilon^{e}$ and a plastic strain $\varepsilon^{p}$. The plastic strain is related to the history of irreversible dissipative processes to which the material was submitted based on three fundamental axioms: the plasticity criterion, the flow rule, and hardening law.

- Yield Criterion. Describes the transition between the elastic and plastic domains through a plasticity function $\Phi$, assuming non-positive values if the stress state is elastic and null values if the stress state reaches its plastic limit.

- The plastic flow rule. The plastic flow rule is defined by a plastic potential function $\Psi$, which specifies how the plastic deformation tensor $\varepsilon^{p}$ evolves in a plasticity process $\dot{\boldsymbol{\varepsilon}}^{p}=\dot{\gamma} \mathbf{N}$, in which $\mathbf{N}=\partial \Psi / \partial \boldsymbol{\sigma}$ is the flow direction.

- Hardening law. Specifies how the internal damage variable evolves $\dot{\boldsymbol{\alpha}}=\dot{\gamma} \mathbf{H}$, where $\mathbf{H}$ is defined as the hardening modulus.

Following [2], the integration procedure to go from a time instant $t^{n}$ to the next one $t^{n+1}$ is usually performed by two major steps. The first step is a purely elastic predictor process (elastic trial step), with $\Delta \gamma=0$. The trial elastic strain and internal variables are defined as $\varepsilon_{\text {trial }}^{e}=\varepsilon^{e, n}+\Delta \varepsilon$ and $\boldsymbol{\alpha}_{\text {trial }}=\boldsymbol{\alpha}^{n} . \boldsymbol{\sigma}_{\text {trial }}$ is calculated according to $\varepsilon_{\text {trial }}^{e}$, and the corresponding $\Phi\left(\boldsymbol{\sigma}_{\text {trial }}, \mathbf{A}\right)$ is computed. If $\Phi\left(\boldsymbol{\sigma}_{\text {trial }}, \mathbf{A}\right) \leq 0$, a valid solution 
to the system is reached, and the variables are updated by the trial values. Otherwise, a plastic corrector step (also known as plastic return-mapping scheme) is performed reformulating the incremental problem solving for $\varepsilon^{e, n+1}, \boldsymbol{\alpha}^{n+1}$ and $\Delta \gamma$ satisfying

$$
\begin{aligned}
\varepsilon^{e, n+1}= & \varepsilon_{\text {trial }}^{e}-\Delta \gamma \boldsymbol{N}\left(\boldsymbol{\sigma}^{n+1}, \mathbf{A}\right) \\
\boldsymbol{\alpha}^{n+1}= & \boldsymbol{\alpha}_{\text {trial }}+\Delta \gamma \boldsymbol{H}\left(\boldsymbol{\sigma}^{n+1}, \mathbf{A}\right) \\
\Delta \gamma>0, \quad & \Phi\left(\boldsymbol{\sigma}^{n+1}, \mathbf{A}\right)=0
\end{aligned}
$$

Next, the plastic strain is updated

$$
\varepsilon^{p, n+1}=\varepsilon^{p, n}+\Delta \varepsilon-\Delta \varepsilon^{e} .
$$

\section{A.1 DiMaggio-Sandler plastic model}

The plastic model adopted in the present study is the DiMaggio-Sandler model [6]. Its plasticity function $\Phi$ is piecewise defined by the yield function $F_{1}\left(I_{1}, \sqrt{J_{2}}\right)$, and by the cap elliptical function $F_{2}\left(I_{1}, \sqrt{J_{2}}, L\right)$, where $I_{1}$ and $J_{2}$ are the first invariant of the stress tensor and the second invariant of the deviatoric stress tensor, respectively, and $L$ is a parameter whose value is generally negative. Precisely,

$$
\Phi\left(I_{1}, \sqrt{J_{2}}, L\right)= \begin{cases}F_{1}\left(I_{1}, \sqrt{J_{2}}\right), & I_{1}>L \\ F_{2}\left(I_{1}, \sqrt{J_{2}}, L\right), & L \geq I_{1} \geq X,\end{cases}
$$

where

$$
\begin{gathered}
F_{1}\left(I_{1}, \sqrt{J_{2}}\right)=\sqrt{J_{2}}-F_{f}\left(I_{1}\right), \\
F_{2}\left(I_{1}, \sqrt{J_{2}}, L\right)=\left(\frac{I_{1}-L}{R F_{f}(L)}\right)^{2}+\left(\frac{\sqrt{J_{2}}}{F_{f}(L)}\right)^{2}-1,
\end{gathered}
$$

and $F_{f}(s)=A-C \exp (B s)$. A typical 2D profile of the yield surface is plotted in Figure 12. Note that $|L|$ specifies the distance of the origin to the center of the ellipse representing the cap of the yield surface.

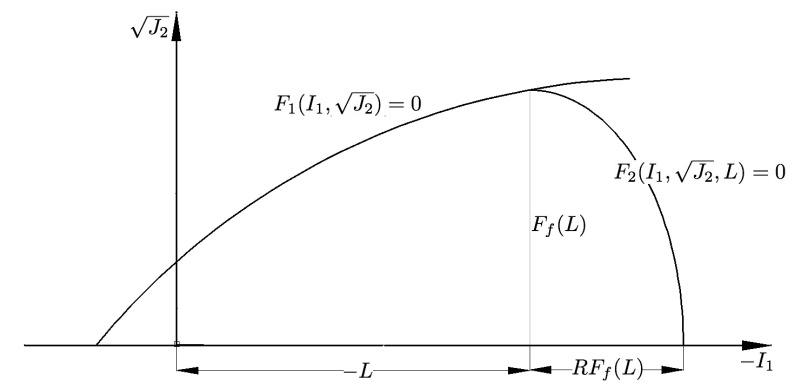

Figure 12: Original DiMaggio-Sandler yield profile in the $\left(I_{1}, \sqrt{J_{2}}\right)$ plane.

The relation between the value of $L$ and the plastic volumetric strain $\epsilon_{v}^{p}$ is defined by:

$$
\varepsilon_{v}^{p}=W[\exp (D X)-1]
$$

where

$$
X=L-R F_{f}(L) .
$$

The constants $A, B, C, D, R$ and $W$ are material properties, which need to be obtained from laboratory tests. 
The DiMaggio-Sandler model is associative such that $\Psi=\Phi$. The corresponding hardening modulus $\mathbf{H}$, and the flow direction $\mathbf{N}$ are related by

$$
\mathbf{H}=\operatorname{tr}(\mathbf{N}) \text {. }
$$

Using this property, the equation for the damage variable at the plastic correction step can be reformulated as

$$
\dot{\boldsymbol{\alpha}}=\dot{\gamma} \operatorname{tr}(\mathbf{N})=\operatorname{tr}\left(\dot{\varepsilon}^{p}\right)=\dot{\varepsilon}_{v}^{p} .
$$

In terms of its Haigh-Westergaard stress representation $\sigma^{*}=\left[\sigma_{1}^{*}, \sigma_{2}^{*}, \sigma_{3}^{*}\right]^{T}$, the yield surface is $\sigma_{1}^{*}$-axially oriented and is formed by two parts: one perfectly plastic surface $S_{1}^{*}$, where $F_{1}=0$, and a cap $S_{2}^{*}(L)$, corresponding to $F_{2}=0$, as represented graphically in Figure 13 . The plastic return-mapping considers these two cases, and the return-mapping scheme is reduced to the resolution of a single non-linear equation.

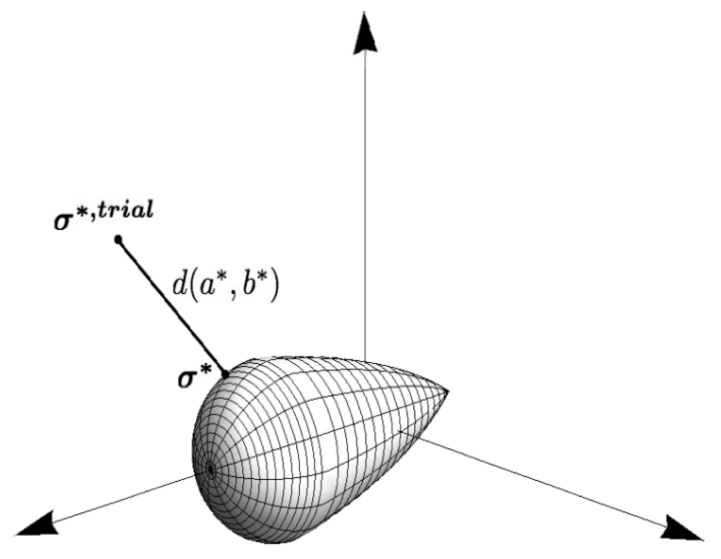

Figure 13: Yield surface of the DiMaggio-Sandler model in the Haigh-Westergaard stress representation. 